\title{
Re-purposing Google Maps Visualisation for Teaching Logistics Systems
}

\author{
France Cheong, Christopher Cheong, and Ferry Jie \\ School of Business IT and Logistics, RMIT University, Australia
}

\author{
france.cheong@rmit.edu.au, christopher.cheong@rmit.edu.au, \\ ferry.jie@rmit.edu.au
}

\begin{abstract}
Routing is the process of selecting appropriate paths and ordering waypoints in a network. It plays an important part in logistics and supply chain management as choosing the optimal route can minimise distribution costs. Routing optimisation, however, is a difficult problem to solve and computer software is often used to determine the best route. In an Australian postgraduate course on logistics systems, Logware, a dated software packaged with a textbook was used for teaching routing. A need was felt to replace the software with a more modern tool with an advanced graphical user interface and a high level of visualisation capabilities for a more engaging student experience and better learning outcomes.

Our objective in this paper is to re-purpose Google Maps, a general mapping utility, and capitalise on its highly visual nature to create a specialised utility for understanding routing and other GIS concepts such as geocoding and geolocation as used in logistics. The re-purposed utility tool was embedded in a teaching method that focused on visualization as a cognitive strategy and took advantage of the human cognitive architecture. Students were trained using a scaffolding approach in which the complexities of the tool developed were increasingly revealed to the students.

The teaching method was used and evaluated in a single 2-hour workshop using mixed-methods research techniques, and positive student experience and learning outcomes resulted. Learning outcomes were measured by assessing students' workshop submission and all students received marks in the 7-10 (out of 10) range. Positive student experience also resulted since 93\% of students rated the "usefulness" of the tool between $7-10$ on a 10 -point scale and $87 \%$ rated "use at work" between 7-10 on a 10-point scale. Positive experience was further evidenced by the qualitative comments students made.
\end{abstract}

Although the study has demonstrated the usefulness of the tool for improving student experience and learning outcomes, one limitation of the study is that the impact of the tools on students' long term retention of learned information was not evaluated. More work needs to be done to evaluate the usefulness of the tool and teaching method over a longer period of time.

Material published as part of this publication, either on-line or in print, is copyrighted by the Informing Science Institute. Permission to make digital or paper copy of part or all of these works for personal or classroom use is granted without fee provided that the copies are not made or distributed for profit or commercial advantage AND that copies 1) bear this notice in full and 2) give the full citation on the first page. It is permissible to abstract these works so long as credit is given. To copy in all other cases or to republish or to post on a server or to redistribute to lists requires specific permission and payment of a fee. Contact Publisher@InformingScience.org to request redistribution permission.
Keywords: visualisation, cognitive learning strategies, Google Maps, logistics

\section{Introduction}

A supply chain consists of activities required for transforming natural resources, raw materials, and components into a finished product for delivery to a customer. Managing a supply chain re- 
quires managing the network of businesses involved in providing goods and services to customers and encompasses the management of all movement and storage of raw materials, work-inprogress inventory, and finished goods from the point of origin to the point of consumption (Harland, 1996). On the other hand, logistics is the management of the flow of goods between the point of origin and the point of destination. Logistics can be considered as a channel of the supply chain that adds time-value and place-utility. It consists of planning, implementing, and controlling the effective level of flows and the storage of goods and services (Ballou, 2007; Bowersox, Closs, \& Cooper, 2010; Coyle, Langley, Gibson, Novack, \& Bardi, 2008; Mentzer, Flint, \& Hult, 2001). One important concept used in logistics and supply chain management is routing, which is the process of selecting paths in a transportation network for distributing goods and services. Routing has implications for the cost effectiveness and efficiency of distribution systems since it is important to select the best route (in terms of distance travelled and/or time taken) in order to minimise transportation costs and provide on-time deliveries.

Finding the best route, also known as the travelling salesman problem, is a complex problem to solve since the number of potential solutions to investigate increases exponentially with the number of intermediate destinations (or waypoints). Since it is impractical or even infeasible to manually determine the best route for many waypoints, a number of computer-based solutions have been developed.

In a postgraduate course in Logistics System offered by an Australian university, with an intake of 70 to 80 students per semester, students are taught routing concepts using Logware, a software packaged with the textbook in use (Ballou, 2004). The latest version of the textbook is the 2004 edition while the software dates back to 1998. Logware is a collection of software programs useful for analysing logistics and supply chain problems and case studies. Its ROUTE module is used to determine the shortest path through a network of routes.

Perceived problems when using Logware in the course include:

- Case studies and data provided with the software are from the United States and cannot be customised to provide an Australian context as desired by students. Since some students may not have a good grasp of US geography, particularly locations, distances, and measurement units (miles vs. kms), an Australian context would provide familiarity and reduce cognitive load.

- Students find the steps to use the software to be quite complicated.

- Level of visualisation is not as sophisticated compared to popular and freely-available modern mapping software.

- Logware operates in a stand-alone mode on a personal computer and is not available online as are many contemporary tools.

Visualisation techniques are most frequently used in the presentation of information and, although they are also powerful problem-solving tools, they are rarely promoted as such in learning and instruction (Rieber, 1995). The emphasis of educational institutions has always been more on verbal, abstract thinking rather than visual and concrete reasoning despite the fact that people have the natural tendency to use visualisation as cognitive strategies. The dominant role of alphanumeric encoding skills in classrooms and textbooks and the subordinate role of visual-spatial learning is a criticism of the current practice in education (Mathewson, 1999). Although a basic goal of education is the successful integration of skills and attitudes into a cohesive mental schema, the current educational system is criticised as this aspect may be absent. Furthermore, with the increasing computational and graphical capabilities of computers (and the availability of a wide range of multimedia software), which are well equipped to implement a range of visualisation strategies, more should be done to devise visual instructional techniques. Exposing students 
to such techniques will inevitably enhance their learning as people are inherently good at interpreting visual patterns.

Google Maps (maps.google.com) is a modern, popular, and freely-available map-based online service provided by Google. Services offered include street maps, which are highly visual diagrams, images of streets and landmarks, and route planning, which provides directions when travelling to multiple destinations. Google also makes available a wide range of Javascript-based application programming interfaces (APIs) (code.google.com/apis/maps) to allow embedding of Google Maps in external websites and overlaying data on top of maps.

Google Maps is commonly used to display given addresses on a map and provide visual directions for travelling to one or more destination addresses. Given the inherent highly visual nature of Google maps, our objective in this study is to re-purpose Google Maps from a general mapping utility for looking up addresses and providing directions to a specialized utility for understanding routing and other GIS concepts such as geocoding and geolocation as used in logistics. The repurposed utility tool was embedded in a teaching method that focused on visualization as a cognitive strategy and took advantage of the human cognitive architecture.

\section{Literature Review}

Visualisation can be broadly considered synonymous to imagery and can be defined as "representations of information consisting of spatial, non-arbitrary, and continuous characteristics" (Rieber, 1995). The picture-like qualities of a visualisation are non-arbitrary because the visualisation resembles the actual object or event, while the continuous characteristics allow the ability of randomly scanning the visualisation in contrast to reading a sequential description of the object.

Visualisation is a cognitive strategy used by human beings for creativity, discovery and problem solving. It has been used by famous people (e.g., Albert Einstein, August Kekulé) to solve complex problems and make scientific discoveries in areas including physics and aviation, and it is also used by ordinary people to solve everyday problems (Rieber, 1995). Cognitive strategies are ways in which people manage their own learning, especially during the problem-solving phase (Weinstein \& Mayer, 1986), and people have a natural tendency to use visualisation as a cognitive strategy. Given this natural tendency, educators are encouraged to consider innovative visualisation strategies to nurture the creative problem-solving process (Rieber, 1995). Like any cognitive process, visualisation is greatly influenced by prior knowledge held in the long-term memory of the individual.

When designing instruction, knowledge of the human cognitive architecture and the structure of the information to be learned can provide useful insights for a successful design (Sweller, 2002). Human cognitive architecture consists of a working memory of limited capacity and duration and a long-term memory of infinite capacity. Working memory is believed to consist of multiple channels or processors, and the visual and auditory channels of working memory are partially separate. The partial separation of the visual and auditory channels can be exploited for learning purposes to increase the capacity of working memory by presenting learning materials in both the auditory and visual modes rather than in a single modality.

In order to learn new material, all the elements of the material must be placed in working memory. If the information structure consists of elements with little or no interactivity between them, then each element can be loaded and learned serially in working memory. However, if element interactivity is high, all the elements must be processed simultaneously in working memory, imposing a heavy cognitive workload. If the new material is sufficiently complex, it cannot be processed adequately in working memory and the learner might fail to understand such material. Other cognitive structures and other learning mechanism are necessary, namely long-term memory and schemas. 
Long-term memory holds well-learned material and is not just used to recognise or recall information but rather as an integral component of all cognitive activities. It is of critical importance to higher cognitive functioning such as problem solving and the problem solving skills depend on the schemas held in long-term memory (De Groot, 1965).

Schemas are categorised information stored in long-term memory to facilitate the processing of materials with high element interactivity in working memory. They allow working memory to treat the many interacting elements as a single stream and without strain. Solving complex problems require the acquisition of thousands of domain-specific schemas (Chi, Glaser, \& Rees, 1982).

Another aspect of the human cognitive architecture is the automation of schemas. After extensive learning practice, well-learned, high element interactivity materials are incorporated into automated schema that can be used to process certain categories of information without much effort on working memory (Kotovsky, Hayes, \& Simon, 1985). As an alternative to practice, another way to achieve automation of schemas is for the learner to mentally visualise the procedures in working memory and, hence, reinforce them in long term memory (Cooper, Tindall-Ford, Chandler, \& Sweller, 2001).

Given the usefulness of visualisation as a cognitive strategy for problem-solving and learning, it has been incorporated in the teaching of courses in various areas. Some examples include: formal reasoning (Oliver \& Aczel, 2002), theoretical computer science (Chudá, 2007), and earth science (Harrower, MacEachren, \& Griffin, 2000). However, regarding using Google Maps and its API as a visualisation technique for enhancing learning, little is reported in the literature as far as we are aware.

\section{Approach}

Our approach for obtaining a more engaging experience and better learning outcomes for students of the Logistics Systems course is by re-purposing Google Maps into a specialised mapping utility (or tool) and embedding the tool in a teaching method with a focus on visualization as a cognitive strategy and taking advantage of the human cognitive architecture. Thus, the mapping utility and the teaching method were centered on three main theories from the relevant literature: visualization, human cognitive architecture, and information structure. Given the visual nature of maps and routing, visualisation was an obvious theory to use as a foundation in our work. Visualisation is a cognitive strategy used for creativity, discovery, and problem solving. We also took advantage of knowledge of the human cognitive architecture and the structure of the information to be learned to guide the design of appropriate learning instructions.

The re-purposed mapping utility was implemented as a web-based software tool for the Logistics Systems course. The tool was integrated with a larger project website named, "Simulation in Learning and Teaching” (SiLT), for easy access. In addition to the software tool, the website also contained general information about the research study and a feedback page. Students were trained to use the site and software tool as part of an interactive 2-hour workshop in which they were also required to carry out assessment tasks. Students' assessment submissions and opinions on the software tool were collected for analysis.

In the following sections, we discuss how we re-purposed Google Maps and how we embedded the re-purposed utility tool in a teaching method. We also discuss how we collected data for evaluating the usefulness of the tool and teaching method. 


\section{Mapping Software Tool}

As many of the students in the logistics systems course do not have high competency with IT, we developed a web-based software tool, which was presented to students in three parts, with each part increasing in functionality and complexity. We believed that this scaffolding and web-based approach would accelerate learning by presenting students with new information that could easily fit into their working memory. Additionally, the tool developed was a mapping tool based on Google Maps, which we believed many students would have some familiarity with and would further assist in accelerating learning as it provides a link to students' long-term memory (for students who have familiarity with Google Maps). Thus, the design of the tool takes into consideration the human cognitive architecture.

A particular benefit inherited from Google Maps is that the tool naturally focuses on visualization and is interactive, which increases user-friendliness and can aid comprehension, especially spatial understanding.

The mapping software tool customised functionality from Google Maps for the purpose of the logistics systems course. The tool is well integrated into the SiLT website and appears as a series of interactive web pages. The tool contains three incrementally complex features: geocoding, geolocation, and routing.

Geocoding is a functionality that translates a given address to a set of latitude and longitude coordinates (a geocode). In our particular implementation, the user is able to enter a series of addresses (refer to Figure 1) and not only does the mapping tool display the geocodes, it also uses Google Maps to convert the addresses to geocodes and displays them on an interactive map (refer to Figure 2). The interactivity of the map is another feature inherited from Google Maps. It allows users to zoom in and out of the map to view different levels of details, and to also navigate to various places on the map, thus adding an element of exploration and discovery.

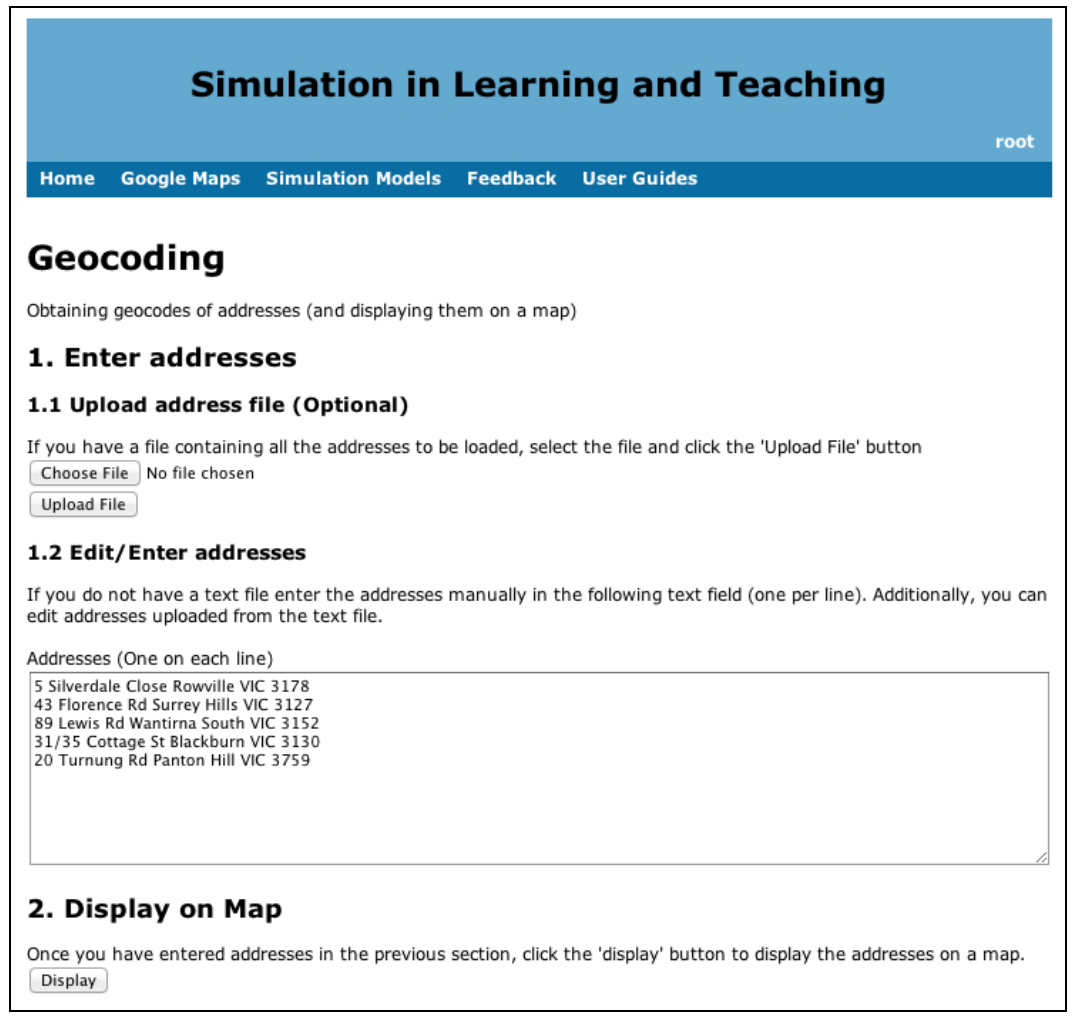

Figure 1. Geocoding Input Page 


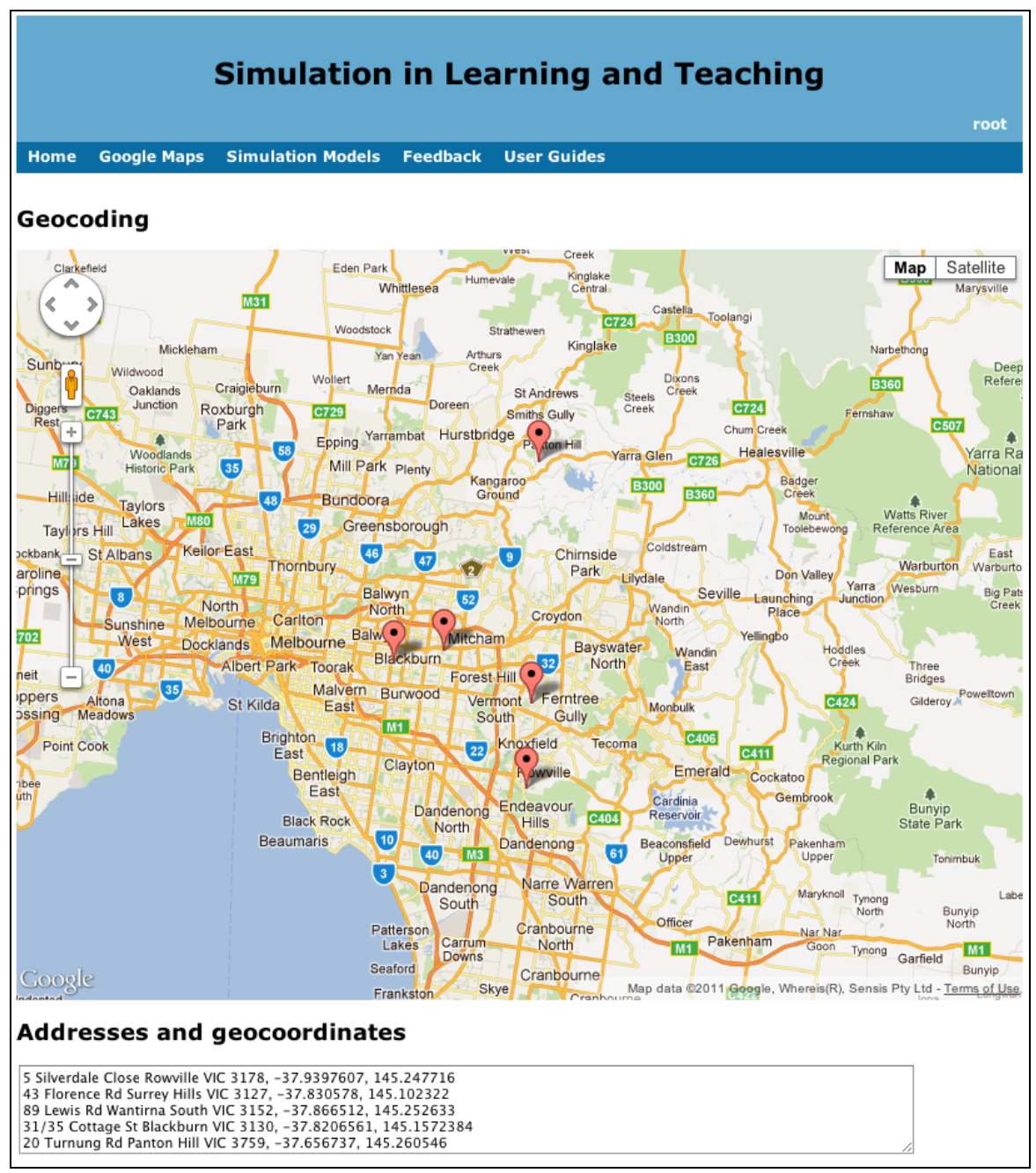

Figure 2. Geocoding Output Page

As an added user-friendly feature, our implementation loads a number of default addresses, so the first-time user does not need to enter any input and can simply press the "Display" button to see the geocoding functionality in action. Thus, there is little commitment from the first-time user in order to see the functionality of the system. The user focuses on the main functionality at hand with little distractors. This reduces the cognitive load on the user's working memory.

For more experienced users (who have understood the functionality of the system and have transferred it to their long-term memory), our implementation allows a text file of street addresses to be loaded. This is a convenience feature that regular users may find useful.

To be precise, geocoding is only the translation of addresses to geocodes. The location of addresses on a map is actually called geolocation. Thus, the first feature of the tool begins with geocoding and transitions users to geolocation seamlessly. Once students have committed the understanding of geocoding to their long-term memory, this also provides a link from that to the new information being held in their working memory, i.e., geolocation.

Geolocation is in fact the next feature of the tool and is slightly more complex. The input screen requires users to enter two sets of addresses: one for all the stores of a company, the other for a number of customer addresses (refer to Figure 3). As with the geocoding feature, default values 
are loaded for the convenience of first-time users and there is also the option to load the addresses from two files (one for store addresses, the other for customer addresses) for experienced users.

Once users submit both sets of addresses, the geolocation feature will display the addresses on a Google Map (refer to Figure 4). Customer addresses are marked in green while store addresses are marked in red. Similarly to the geocoding feature, the map contains interactive features from Google Maps such as zoom in and out, and navigating to various places on the map. Additionally, for convenience and added interactivity, our implementation provides users with the ability to toggle (show/hide) the display of the customers and stores on the map.

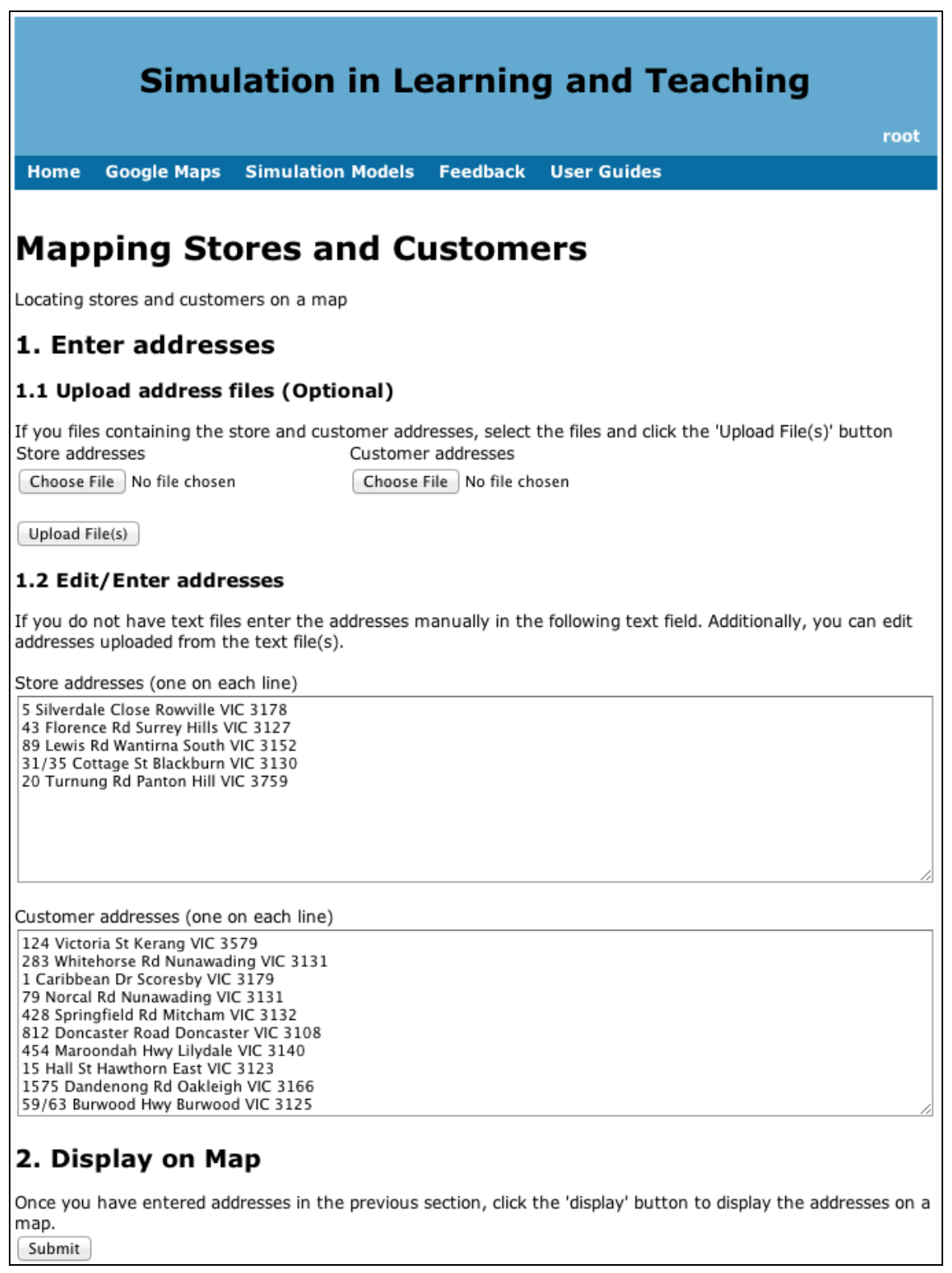

Figure 3. Geolocation Input Page 


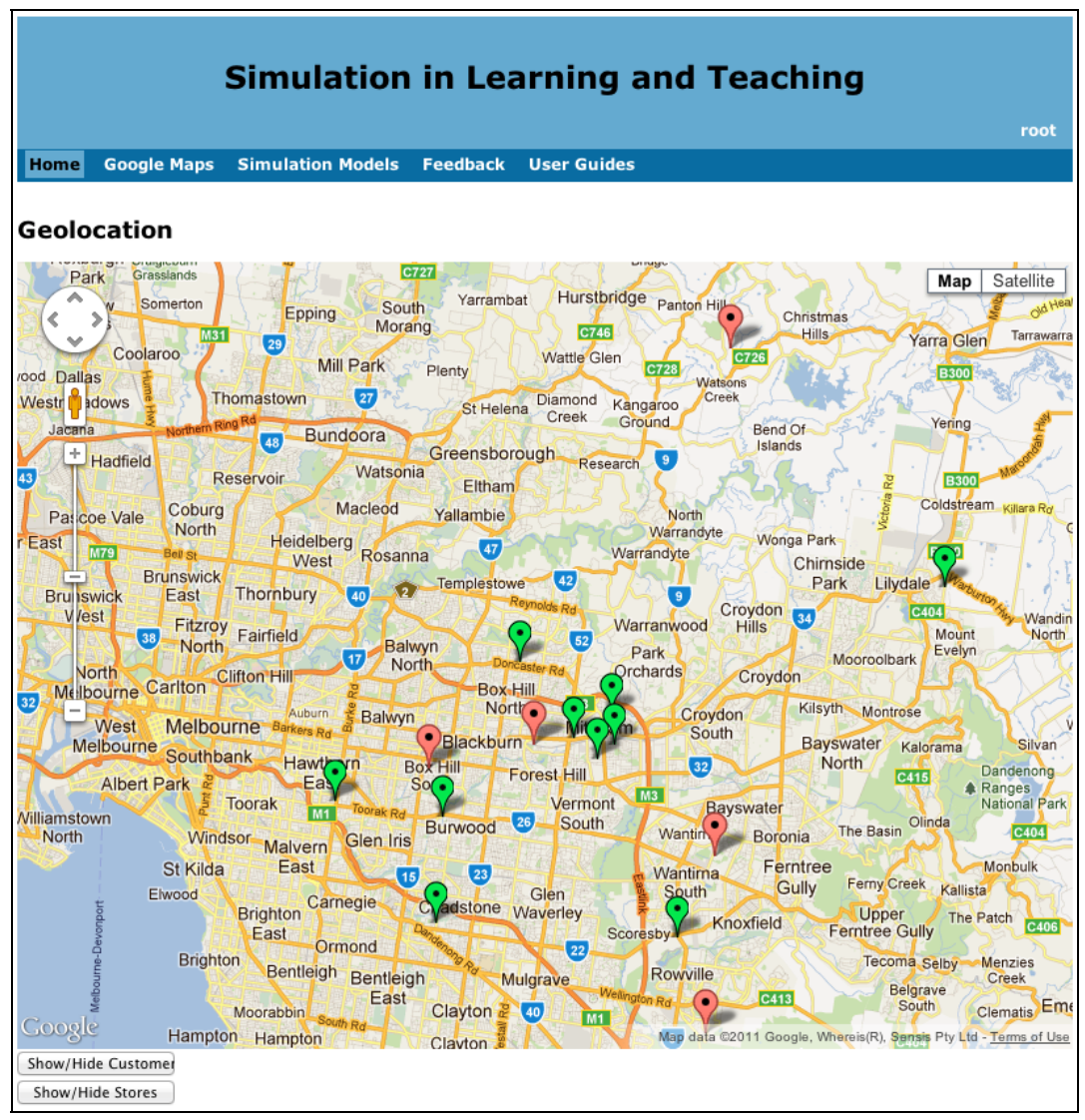

Figure 4. Geolocation Output Page

The last feature of the mapping tool is the most complex. It plans a route and requires the user to enter three distinct sets of data: (1) a starting address, (2) a set of destination addresses to visit (called "waypoints"), and (3) a final destination address (refer to Figure 5). As with the previous two features of the mapping tool, default addresses are loaded, as a convenience for first-time users. An additional input is for users to select is the "optimise waypoints" option. When selected, the mapping tool will use Google Map's routing optimisation feature to optimise the waypoints by both distance and time.

When users submit their inputs, the addresses are marked on a Google Map in visitation order (optimised if the "optimise waypoints" option is selected or in order entered by user if not selected) with the suggested routes between the addresses marked (refer to Figure 6). Our implementation also provides a table to display information such as distance and time travelled between each segment of the trip, and the overall distance and time travelled for the entire trip.

A natural response from first-time users is to try the routing feature without optimisation first and then try it again with it (or vice versa) to see the difference. Of course, the default data loaded in the system takes advantage of this natural curiosity to show a clear difference between optimised and non-optimised routing. 


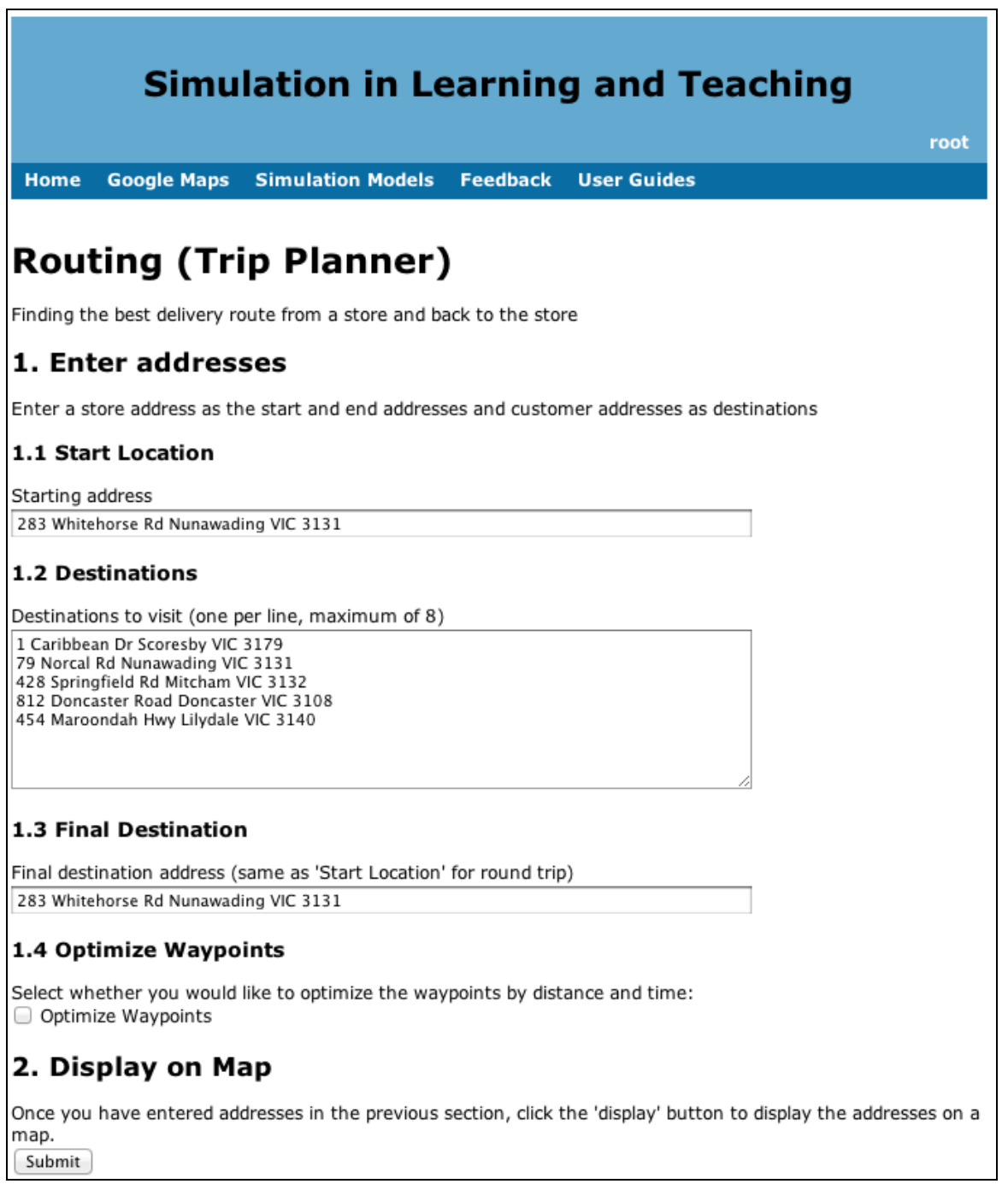

Figure 5. Routing Input Page 


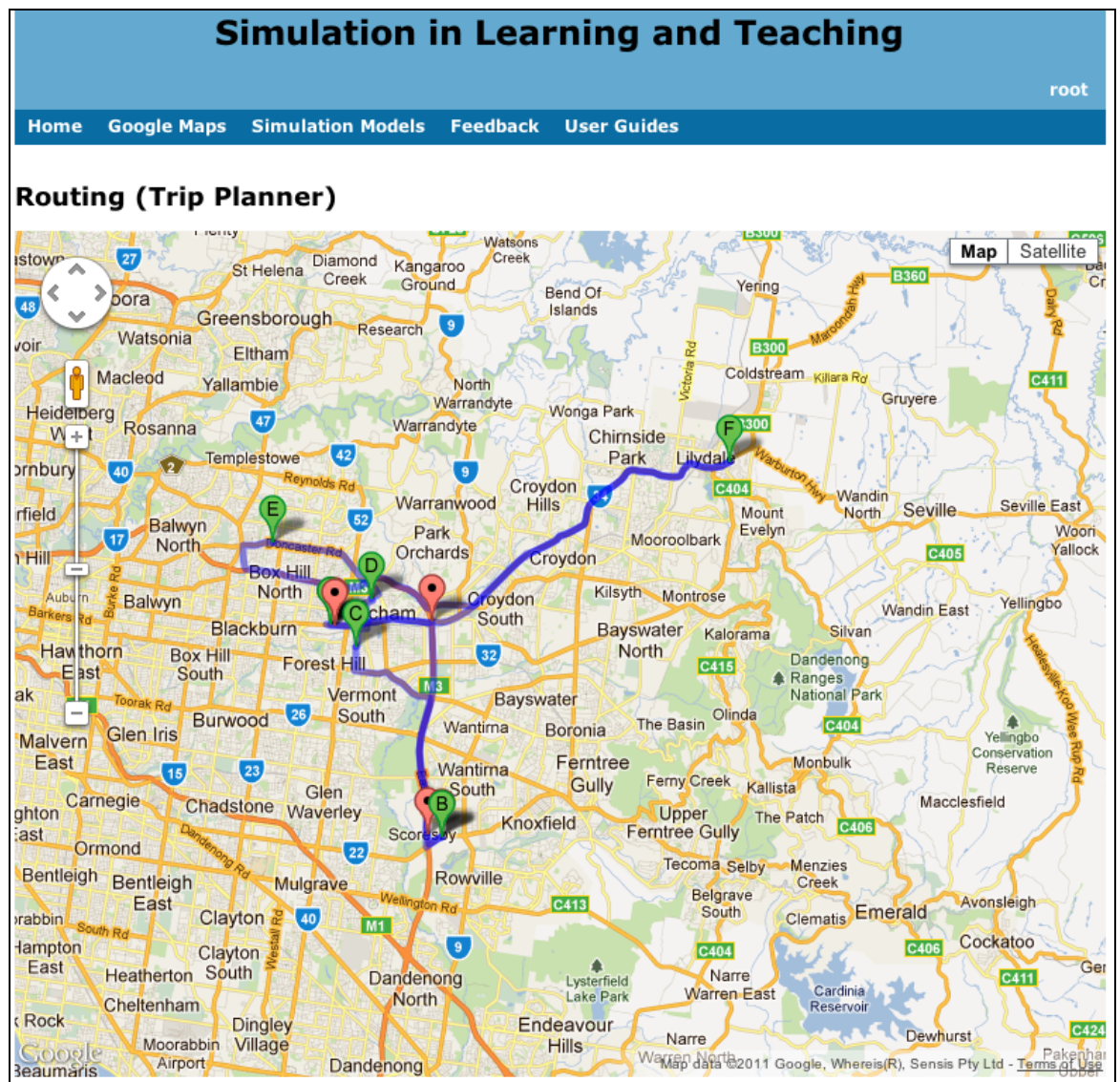

Route Segments

\begin{tabular}{|c|c|c|c|c|}
\hline Segment & Start & End & Distance & Time \\
\hline $1(A-B)$ & $\begin{array}{l}283 \text { Whitehorse Rd, Nunawading VIC } 3131 \text {, } \\
\text { Australia }\end{array}$ & $\begin{array}{l}1270 \text { Ferntree Gully Rd, Scoresby VIC } 3179, \\
\text { Australia }\end{array}$ & $14.7 \mathrm{~km}$ & $17 \mathrm{mins}$ \\
\hline $2(B-C)$ & $\begin{array}{l}1270 \text { Ferntree Gully Rd, Scoresby VIC } 3179 \text {, } \\
\text { Australia }\end{array}$ & 79 Norcal Rd, Nunawading VIC 3131, Australia & $11.9 \mathrm{~km}$ & 14 mins \\
\hline $3(C-D)$ & 79 Norcal Rd, Nunawading VIC 3131, Australia & 1 Venice Ave, Mitcham VIC 3132, Australia & $2.8 \mathrm{~km}$ & $6 \mathrm{mins}$ \\
\hline $4(D-E)$ & 1 Venice Ave, Mitcham VIC 3132 , Australia & $\begin{array}{l}812 \text { Doncaster Rd, Doncaster VIC } 3108, \\
\text { Australia }\end{array}$ & $5.7 \mathrm{~km}$ & $9 \mathrm{mins}$ \\
\hline $5(E-F)$ & $\begin{array}{l}812 \text { Doncaster Rd, Doncaster VIC 3108, } \\
\text { Australia }\end{array}$ & $\begin{array}{l}\text { 450-452 Maroondah Hwy, Lilydale VIC } 3140, \\
\text { Australia }\end{array}$ & $26.8 \mathrm{~km}$ & $29 \mathrm{mins}$ \\
\hline $6(F-G)$ & $\begin{array}{l}\text { 450-452 Maroondah Hwy, Lilydale VIC } 3140 \text {, } \\
\text { Australia }\end{array}$ & $\begin{array}{l}283 \text { Whitehorse Rd, Nunawading VIC } 3131 \text {, } \\
\text { Australia }\end{array}$ & $20.5 \mathrm{~km}$ & $28 \mathrm{mins}$ \\
\hline Total & & & 82.5 km & $\begin{array}{r}103 \\
\operatorname{mins}\end{array}$ \\
\hline
\end{tabular}

Figure 6. Routing Output Page

\section{Teaching Method}

Students were trained to use all three of the mapping software tool features in a workshop. The workshop was conducted in interactive mode by two of the researchers and scaffolding techniques were used to help students successfully complete assigned tasks so that they can quickly progress to the next ones. The workshop was carefully planned in advance, and it was divided into a number of tasks with progressive levels of difficulty. For each task, tutors interacted with students by means of demonstrations, questioning techniques, identifying hints, and cues, and they circulated among students to question their approach to the task and provide students with constructive feedback. As part of the workshop, the students were required to complete exercises, which were collected, assessed (for academic purposes), and analysed (for research purposes).

The mapping software tool was designed to reveal features in increasing complexity, and thus, the training workshop was also designed using this scaffolding approach. The aim of the workshop is 
for students to gain an appreciation of the difficulty of manually determining the best route and to also gain technical skills in using the mapping software tool developed to easily determine the best route.

The workshop began by introducing the students to a definition of routing and its application and usefulness in logistics and supply chain management (minimising distance and time travelled, and thus increasing cost effectiveness and efficiency of distribution systems). This also provided the students a preview of what their ultimate task in the workshop would be and motivated their interest.

The next part of the workshop briefly described the difficulties of routing (the number of potential solutions increases exponentially with each additional waypoint) and highlighted that it is impractical and, in some cases, infeasible to determine the best route manually.

Students were informed of the aim of the workshop and presented with their first task. To appreciate the difficulty of determining the best route, the instructor demonstrated how to manually map a given set of addresses using Google Maps (the publicly available version and not the mapping software tool developed) and how to change the sequences of the waypoints to alter distance and time travelled.

Demonstration of the operation of the highly visual software and tasks to be performed using verbal explanations and instructions is an important part of the process of using the learning tool as this takes advantage of the fact that working memory has partially separate visual and auditory processing capabilities. In effect, we are trying to increase the capacity of working memory and hence speeding up the learning of complex concepts.

For their first task, students were challenged to find and record the best route they could find. After 20 minutes, the instructor asked students to volunteer their answers until the best route found in the class was determined.

For the assessment of this task, students were asked to individually answer the following questions:

1. What do you mean by best sequence (i.e., route)?

2. Are there any alternative best sequences? If so, what are they?

3. Can you think of another scenario where you would use the best route? Explain your scenario and its usefulness.

Once students appreciated the difficulty of finding the best route using the basic functionalities of Google Maps available to the general public, they were given their second task. The instructor introduced the SiLT website and demonstrated the three features (geocoding, geolocation, and routing) of the mapping software tool. In order to facilitate the tasks of students, the mapping software tool automated a large part of the mapping tasks involved such that students could focus on the task at hand rather than using trial-and-error techniques to arrive at a solution. Students were then asked to determine the best route using the routing feature of the mapping software tool and to answer the following questions as part of their assessment:

1. Briefly explain the results generated by the SiLT routing tool.

2. Evaluate the usefulness of the SiLT tool by comparing and contrasting the solution generated with your previous manual routing response using Google Maps. Hint: consider the differences on the following dimensions, sequence of destinations, travelling distance, travelling time, effort required to determine solution.

3. Do you find the [mapping] tool useful for facilitating your learning in this workshop? (1 = Not useful, 10 = Very useful). Justify your rating. 
4. Would you use the tool at work? (1 = Unlikely, 10 = Very likely). Justify your rating.

The instructor concluded the workshop by discussing some of the issues that emerged from the tasks and any further questions students may have had.

\section{Data Collection}

At the end of the workshop, workbooks were collected and marked for assessment purposes. To ensure consistency, only one assessor marked all the submissions. Marks were allocated based on the students' understanding of the material. The maximum marks for the entire workshop was 10 , and the workshop was worth $5 \%$ of the total marks for the entire course.

From the collected workbooks, we analysed the responses and the marks attained by the students. In particular, we focused on responses to question 2 as an indicator of understanding the value of the mapping software tool, and questions 3 and 4, as indicators for usefulness and uptake of the mapping software tool, respectively. We also analysed the comments made by students in their workbooks. Since only 22 students out of a cohort of 71 agreed to participate in the research, only the comments made by these students will be discussed in the analysis section. Although the sample size was small, it was fairly representative of the population in terms of gender, age group and mode of study (refer to Table 1).

Table 1: Demographics of surveyed students

\begin{tabular}{|c|c|c|c|c|}
\hline Characteristics & \multicolumn{2}{|c|}{ Population } & \multicolumn{2}{c|}{ Sample } \\
\hline & Count & $\%$ & Count & $\%$ \\
\hline Gender & & & & \\
\hline Male & 36 & $50.7 \%$ & 13 & $59.0 \%$ \\
\hline Female & 35 & $49.3 \%$ & 9 & $41.0 \%$ \\
\hline Age group & & & & \\
\hline$<20$ & 0 & & 0 & \\
\hline $21-28$ & 55 & $77.46 \%$ & 18 & $81.82 \%$ \\
\hline $29-48$ & 14 & $19.72 \%$ & 4 & $18.18 \%$ \\
\hline $49-65$ & 2 & $2.82 \%$ & 0 & \\
\hline$>65$ & 0 & & 0 & \\
\hline Mode of study & & & & \\
\hline Full time & 62 & $87.3 \%$ & 19 & $86.4 \%$ \\
\hline Part time & 9 & $12.7 \%$ & 3 & $13.6 \%$ \\
\hline
\end{tabular}

\section{Results and Discussion}

From the data analysis, descriptive statistics about the three questions asked were calculated, as presented in Table 2. 
Table 2. Descriptive Statistics of Marks Attained, Usefulness, and Use at Work

\begin{tabular}{|l|r|r|r|}
\hline Statistic & Marks & Usefulness & Use at Work \\
\hline Minimum & 7.0 & 3.0 & 1.00 \\
\hline Average & 8.393 & 8.257 & 8.214 \\
\hline Median & 8.0 & 8.0 & 9.0 \\
\hline Mode & 8.0 & 8.0 & 10.0 \\
\hline Max & 10.0 & 10.0 & 10.0 \\
\hline Standard Deviation & 0.737 & 1.612 & 2.180 \\
\hline
\end{tabular}

The overall results were very positive. The statistics show that, in general, most students had a good understanding of the material covered and use of the software mapping tools (marks ranged from a minimum of $70 \%$ to $100 \%$, with an average of $84 \%$, and a low standard deviation of 0.737). The median and mode showed that most students attained a result of $80 \%$.

\section{Learning Outcomes}

A histogram of the marks achieved by the students (refer to Figure 7), reveals that $55 \%$ percent of students received $8 / 10$, $24 \%$ received $9 / 10$, and $17 \%$ received $10 / 10$.

When comparing their manually generated results against the results generated by the mapping software tool (question 2), many students stated that the use of the mapping software tools resulted in a better route and was more effective and efficient. Comments such as, "Easier to get solution, less effort, less time ...", "Easier to use, provided shortest and quicker route”, and "Very useful, no trial and error" were common.

There was some confusion as to the accuracy of the address of locations. As part of its usual operation, Google Maps will approximate the location of an address if it cannot determine it exactly. In some cases, this approximation can be very far from the intended address, and there were some such cases with addresses that students had to use. Some students claimed that the publicly available Google Maps was more accurate while others claimed the opposite; that the mapping software tool was more accurate.

This was an unintended technicality that had both positive and negative aspects. Some students identified it and realised that one should not blindly accept the results generated and that one should double-check to ensure correctness. However, the inaccuracy made some students distrustful of the technology, and they undervalued the usefulness of the tool and simply believed it to be inaccurate as a whole.

Another aspect that reduced the usefulness of the tool for students was that it did not consider "traffic condition, timing, weight of cargo, ...". Those students who made these comments realised that not all factors were considered, but also failed to realise that the tool was a generic routing tool and not fully customized for logistics distributions and, thus, would not naturally consider factors such as cargo weight. This is a common issue with students with low IT literacy; they have high expectations of technology, which can be unrealistic (e.g., that a system will consider each and every real-life factor involved). 


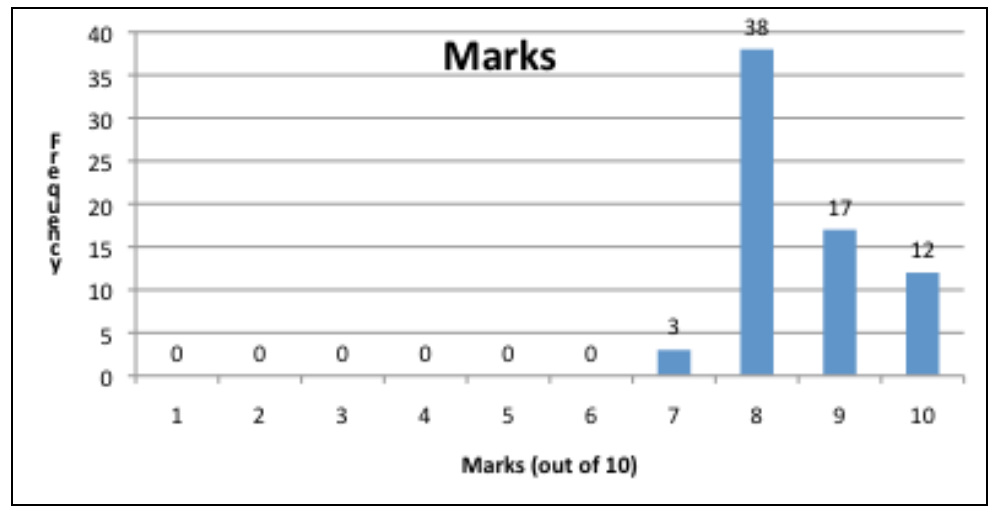

Figure 7. Histogram of Marks Attained

As hinted upon in their results to question 3 , most students found the mapping software tool to be useful. This is supported by the descriptive statistics about their responses to question 3 (refer to Table 2). In general, students found the tool to be useful as the ratings ranged from a minimum of 3 to a maximum of 10 , with a median and mode of 8 . Compared to the marks attained, there is more variability in the usefulness ratings with a standard deviation of 1.612 .

\section{Student Experience}

Inspection of the histogram of usefulness ratings (refer to Figure 8) shows that although there is more variability, the majority of ratings are toward the upper end (93\% are in the 7-10 range) of the usefulness scale.

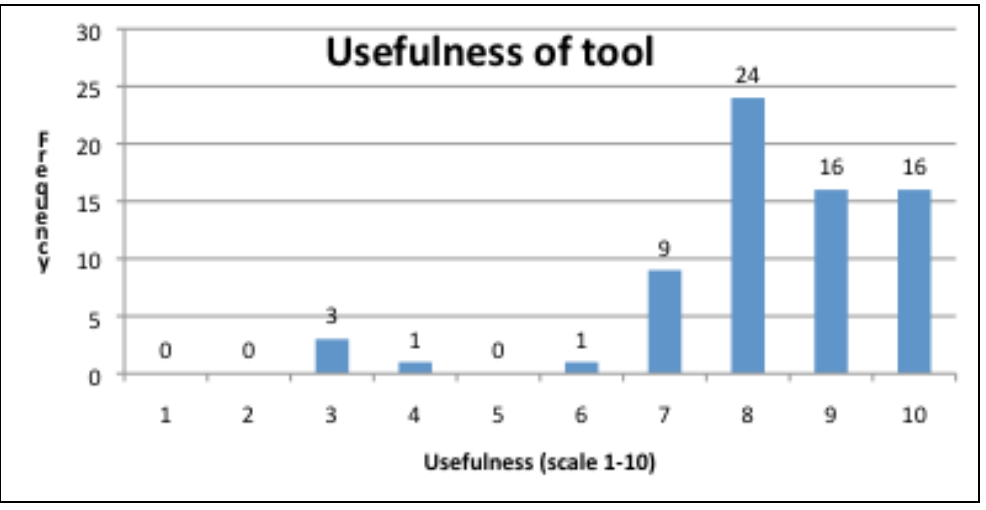

Figure 8. Histogram of Usefulness of Mapping Software Tool

Analysis of the justification for the ratings reveal that the low ratings were attributed to either misconception, "Same as Google Maps!", or distrust of the tool due to lack of details, "Very simple, don't know what's going on behind interface", and "Better to understand the algo[rithm]!”.

Some of the justifications provided by students re-iterated the ease-of-use, effectiveness, and efficiency of the tool, e.g., "Useful, automatic, saves time and little effort", and "User friendly, fast, practical". There are also comments which hint at the visual nature of the tool, "Visual and easy to justify decisions, simple UI", and others that state the tool was easy to learn, "Easy to use, no need for much learning to use tool". Such characteristics of the tool were not always seen as positive, as one student who provided a low rating stated, "Will make us become lazy!" 
There were also re-iterations of the tool not being specific enough for logistics distribution, "Very useful and convenient, but still not perfect", and "Fast and efficient, but does not consider traffic, road blocks, certain days”.

Some students found the technology and approach to be useful, "Yes, shows how theory can be applied and very visual with maps", "Shows how IT is essential instead of manual calculations and errors", and "New logistics technology for me". Other students revealed they were made more aware of issues related to routing and logistics, "Create awareness of choosing optimal route", and "Transportation costs are huge! Helps to understand importance of routing".

Students also realised the links between theory, the workshop, and practice, "Reinforces what [was] learned in [the] workshop", and "Really useful to connect practice and knowledge learned in textbook".

As the course was at postgraduate level and many of the students work, question 4 enquired if they would use such a tool at work as an indicator of future use (they would not use it again in the course). The responses included students who did not work, "Unlikely to use, full time student", and others who would have no use for this type of tool in their positions (but still found it valuable), "Seldom use this kind of tool", and "Not in current position, but can see benefits on delivery and business".

As with the previous answers, there were accuracy concerns, "Saves time, no manual operation, but accuracy is a bit of concern", and concerns about a lack of realism, "Useful for learning, but in real life more factors e.g. cost, traffic condition, etc. [need to be considered]", "Useful but need to consider other factors: toll, rush hour, etc.", "Use approximations, not useful in practice", and "Just a fixed calculation, not flexible enough".

There were also other practical issues raised, such as a lack of deeper integration with other systems, "No ERP integration. No traffic condition! No on the fly adjustment in case of accident", and "Does not arrange transport but will use it and prefer more advanced program, e.g. timing, traffic".

Other justifications pointed out the ease of use, and usefulness of the tool, "Online system, doesn't need setup", "Definitely will, easy to use and does not require much knowledge", and "Yes, have many truckloads with multiple drops". One student even stated that the tool was advanced, "An advanced tool, many advantages when compared with others".

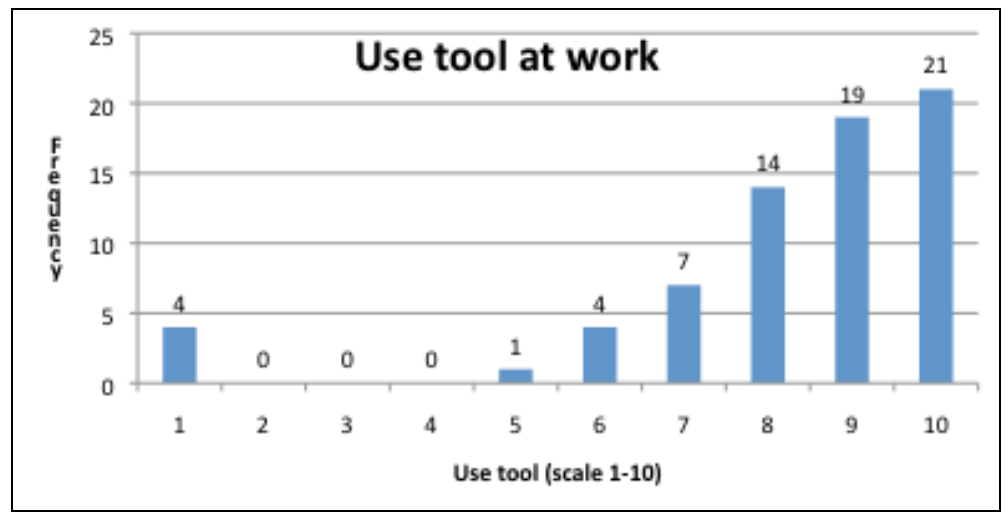

Figure 9. Histogram of Use at Work

Although there was the most variability in this set of responses (a standard deviation of 2.180), most of the responses were higher ratings (a median and mode of 9 and 10, respectively) compared to the other questions. $87 \%$ of the answers were in the 7-10 range. 
The use of scaffolding in the delivery of the workshop and the design of the mapping software tool facilitated accelerated learning. Students were able quickly understand the theory involved and put to practice recently acquired skills in using the tool. Some students were able to attain a higher level of learning by abstracting concepts and applications of the tool while others focused on the limitations of the tool (did not consider all factors, lack of realism), and others had misgivings about the tool due to a lack of details.

On the whole, the results related to learning (as indicated by question 2) are very positive. All students achieved marks between 7-10, with the majority achieving 8/10. The comparison of the tool against the publicly available Google Maps is also positive as many of the students realised the advantages offered by the mapping software tool. Furthermore, many students stated that they would use the tool at work.

A running issue throughout the three questions is the lack of realism or lack of consideration of other factors in the routing algorithm. This is a difficult issue to address as the lack of realism is actually useful in allowing the students to more easily and quickly understand the tool. Inclusion of all relevant factors would probably not have been conducive to the success of the delivery approach as it would have added increased complexity in the students' initial comprehension of the tool. Although it is possible to include more factors in the tools to simulate more realistic operating conditions, this might not be a desirable feature especially in a tool for learning basic routing. Students may have difficulties comprehending the outcome of the complex interactions of a multitude of factors when making routing decisions.

\section{Relating Findings to Theories}

Relating our findings to the theories upon which our work is founded, we can make the following remarks. Students found the mapping tools developed to be easy to use. Even students from nonIT background were not stressed in learning to use the new technology-based tool and some even found it enjoyable. Although the "ease of use" can be largely attributed to the highly visual nature of Google Maps and the technological facilities offered by modern web browsers, the scaffolding techniques used also contributed to "ease of use" to ensure learning occurred. Such scaffolding techniques included tutor interactions with students by means of demonstrations, questioning techniques, circulating among students to question their approach to the task, and providing hints, cues, and constructive feedback.

Students also found that the tools and the workshop made it easy to learn routing concepts. Students understood not only the complex concepts but also mentioned that using technology is a good way to connect theory and practice in this case. Although the "ease of use" aspect probably contributed to "ease of learning" to some extent, the major contributing factor is most likely to be the "specific" scaffolding techniques used in the study. These scaffolding techniques were based on knowledge of the human cognitive architecture and structure of the information to be learned in the workshop. In particular, we broke down the learning of complex routing concepts into related bite-size chunks (such as geolocation, geocoding, manual trial-and-error solutions, and automated solutions) to ensure that new information presented to students fit into their working memory. Furthermore, the Google-Maps-based tools and the instruction methods used in the workshop helped create a learning environment that provided links to students' long term memory in order to facilitate the processing of information held in their working memory.

The results of using an "easy to use" and "easy to learn" teaching tool in the classroom made it easier for students to learn and, hence, they became more open towards learning and they achieved high learning outcomes as found in the study. This is in line with the general belief that the easier we make learning for students, the more willing they will be to learn. Although the ultimate goal of education should be retention of learned materials for future use, we did not test 
this aspect in our study since what was learned during the 2-hour workshop was tested in the same workshop. In order for retention of learning to occur, the learned contents in working memory should be transferred to long-term memory. We have no evidence this was the case as we did test what was learned at a later stage. This is a limitation of the study which will be addressed in future work.

Another possible limitation of our work is that evaluation of learning outcomes was mainly by assessing students' work by one of the investigators involved in the research. Although this might be considered as having introduced some element of bias in the findings of the study, it was ensured that this was done as objectively as possible by using a marking guide. Furthermore, the consistency of the findings of the various elements of the study also support our argument that using an "easy to use" and "easy to learn" tool was an effective way of teaching routing concepts since high learning outcomes were achieved by students.

\section{Implications for Pedagogy}

Essentially, our contribution is the framework we used for successfully integrating technology with instruction methods to teach complex concepts to students of especially non-IT background. During the requirements gathering and analysis stage for building the tool, we considered the fact that users (students as well as their instructor) have low IT background/competency. Since the tool to be developed deals with complex concepts, it will by nature possess some inherent complexities. When dealing with students of non-IT background, another important aspect to consider is the method used to provide them with access to the software. Instead of getting them to download, install, and configure the software, it is better to make the tool available online and for them to log to the web site to gain access. During the design stage, apart from ensuring that the characteristics of the tool to be built matched user requirements, we also designed a matching underlying teaching approach, mainly related to "generic" scaffolding approaches as well as "specific" ones based on human cognitive architecture and information structure in order for bite-size learning to occur. When implementing the tool, we re-purposed highly visual tools such as Google Maps and used web-based technologies as they would provide an element of familiarity even to students of non-IT background.

\section{Conclusion}

Our objective in this paper was to re-purpose Google Maps from a general mapping utility to a specialised mapping tool for teaching complex GIS concepts in a logistics systems course to enhance student experience and learning outcomes. The re-purposed utility tool was used in a 2hour workshop that focused on visualization as a cognitive strategy and took advantage of the human cognitive architecture and information structure. Students were trained in the use of the tools using "generic" scaffolding approaches (demonstrations, questioning techniques, providing hints, cues, and feedback) and "specific" scaffolding approaches based on knowledge of the human cognitive architecture and structure of the information to be learned in the workshop. Specific scaffolding techniques involved breaking the task of learning complex routing concepts into related bite-size tasks such that new information presented to students easily fits into working memory and providing links to long term memory in order to facilitate the assimilation of new information.

Based on usage in a single workshop, the teaching method was evaluated using mixed-methods research techniques. Learning outcomes were measured by assessing students' workshop submission and results showed positive learning outcomes. All students received marks in the 7-10 (out of 10) range. Positive student experience also resulted since $93 \%$ of students rated the "usefulness" of the tool between 7-10 on a 10-point scale and 87\% rated "use at work" between 7-10 (again on a 10-point scale). 
Comments submitted by students were also largely positive. Overall, they found the tool to be easy to use as it was easy to obtain a solution with little effort or time. They also believed the tool to be useful to show how theory can be applied and raised awareness about choosing the optimal route in logistics and distribution/transportation networks.

The contribution of this study is the framework used for successfully integrating technology with instruction methods to teach complex concepts to students, especially those of non-IT background.

Although the results were generally positive, the research was limited to being trialled and evaluated in single workshop. Since there is no evidence that what was learned in the single workshop was committed to long term memory for future use, more work needs to be done to evaluate the usefulness of the tool and teaching method over a longer period of time.

\section{Acknowledgements}

We would like to acknowledge the contributions of Nicholas Potesta, who worked in the School of Business IT and Logistics, RMIT University and assisted in the design and development of SiLT website.

We gratefully acknowledge the funding provided by the Learning and Teaching Investment Fund of RMIT University to execute this project.

The research was conducted with ethics approval from the RMIT Business College Human Ethics Advisory Network under register number 1000121.

\section{References}

Ballou, R. H. (2004). Business logistics/supply chain management: Planning, organising, and controlling the supply chain. Pearson/Prentice Hall.

Ballou, R. H. (2007). The evolution and future of logistic and supply chain management. European Business Review, 19(4), 332-348.

Bowersox, D., Closs, D., \& Cooper, B. (2010). Supply chain logistics management (3rd ed.). NY: McGraw- Hill/ Irwin.

Chi, M., Glaser, R., \& Rees, E. (1982). Expertise in problem solving. In R. Sternberg (Ed.), Advances in the psychology of human intelligence (pp. 7-75). Hillsdale, NJ: Erlbaum.

Chudá, D. (2007). Visualization in education of theoretical computer science. Paper presented at the International Conference on Computer Systems and Technologies - CompSysTech’07.

Cooper, G., Tindall-Ford, S., Chandler, P., \& Sweller, J. (2001). Learning by imagining. Journal of Experimental Psychology: Applied, 7, 68-82.

Coyle, J. J., Langley, C. J., Gibson, B., Novack, R. A., \& Bardi, E. J. (2008). Supply chain management: A logistics perspective. Mason, USA: South-Western.

De Groot, A. (1965). Thought and choice in chess. The Hague, Netherlands: Mouton.

Harland, C. M. (1996). Supply chain management, purchasing and supply management, logistics, vertical integration, materials management and supply chain dynamics. In N. Slack (Ed.), Blackwell Encyclopedic dictionary of operations management. UK: Blackwell.

Harrower, M., MacEachren, A., \& Griffin, A. L. (2000). Developing a geographic visualization tool to support earth science learning. Cartography and Geographic Information Science, 27(4), 279-293.

Kotovsky, K., Hayes, J. R., \& Simon, H. A. (1985). Why are some problems hard? Evidence from Tower of Hanoi. Cognitive Psychology, 17, 248 - 294. 
Mathewson, J. H. (1999). Visual-spatial thinking: An aspect of science overlooked by educators. Science Education, 83(1), 33-54.

Mentzer, J. T., Flint, D. J., \& Hult, G. T. M. (2001). Logistics service quality as a segment-customized process. The Journal of Marketing, 65, 82-104.

Oliver, M., \& Aczel, J. C. (2002). Theoretical models of the role of visualisation in learning formal reasoning. Journal of Interactive Media in Education, 3.

Rieber, L. P. (1995). A historical review of visualization in human cognition. Educational Technology Research and Development, 43(1), 45-56.

Sweller, J. (2002). Visualisation and instructional design. Paper presented at the International workshop on dynamic visualizations and learning, Knowledge Media Research Center, Tubingen, Germany.

Weinstein, C. F., \& Mayer, R. F. (1986). The teaching of learning strategies. In M. C. Wittrock (Ed.), Handbook of research on teaching. New York: Macmillan.

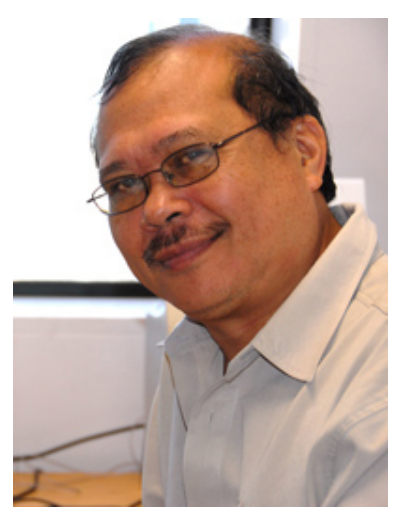

\section{Biographies}

Dr France Cheong is a senior lecturer in the School of Business IT and Logistics, RMIT University. He holds a Bachelor in Business (Computing), a Master of Computer Science, a Master of Education, and a Ph.D. (Computer Systems Engineering).

His research interests include the modelling and simulation of complex systems using a wide range of techniques such as: fuzzy systems, evolutionary computation, artificial immune systems, agent-based modeling and system dynamics. He has applied these techniques to engineering, business applications, and education.

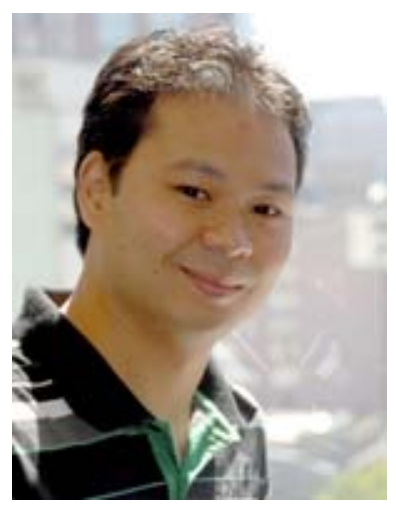

Dr Christopher Cheong is a lecturer in the School of Business IT and Logistics, RMIT University. He holds a Bachelor of Applied Science (Computer Science)(Honours), a Graduate Certificate in Tertiary Teaching and Learning, and a Ph.D. (Computer Science).

His general research interests lies in the areas of artificial intelligence, intelligent agents, evolutionary computing, software engineering, and modeling and simulation. He is interested in applying these to various domains, including education.

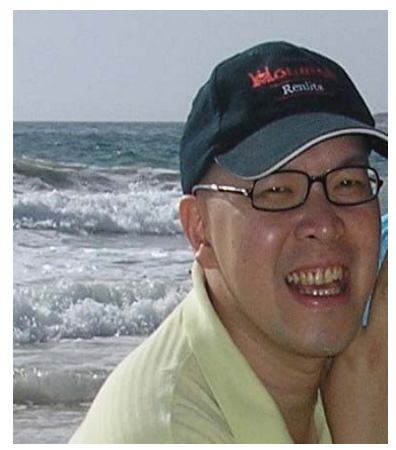

Dr Ferry Jie is a lecturer at School of Business IT and Logistics, RMIT University. He received his doctoral degree from the University of Sydney.

His research interests are supply chain management, operations research, total quality management, lean six sigma, production planning and inventory control, logistics system (including reverse logistics) and strategic management. 